\title{
Categorical Consequence for Paraconsistent Logic
}

\author{
FRED JOHNSON Colorado State University \\ johnsonf@lamar. colostate.edu
}

PETER W. WOODRUFF University of California, Irvine

pwoodrufQuci.edu

\begin{abstract}
Consequence relations over sets of "judgments" are defined by using "overdetermined" as well as "underdetermined" valuations. Some of these relations are shown to be categorical. And generalized soundness and completeness results are given for both multiple and single conclusion categorical consequence relations.
\end{abstract}

Rumfitt [1] discusses multiple-conclusion consequence relations defined over sets whose members, if any, are assertions or rejections. The consequence relations are generated by sets of valuations whose members include those that admit truth value gaps - there are sentences that are neither true nor false on some valuations. Rumfitt shows that the consequence relations are categorical - that is, the consequence relations generated by distinct sets of valuations are distinct. Given Rumfitt's work, it is natural to ask whether categoricity holds for multiple-conclusion consequence relations generated by sets of valuations whose members include those that admit truth value gluts - there are sentences that are both true and false on some valuations. We will show that the answer is Yes.

Johnson [2] extends Rumfitt's work to obtain categoricity results for single conclusion consequence relations generated by valuations that allow truth-value gaps. We will extend his discussion by considering valuations that allow truth-value gluts.

We also will extend Shoesmith and Smiley's [3] generalized soundness and completeness results for consequence relations generated by valuations that admit neither gaps nor gluts to those that do. ${ }^{1}$

\footnotetext{
${ }^{1}$ Our discussion is heavily influenced by Shoesmith and Smiley [3] and Smiley [4]. The latter gives credit to Carnap [5] and [6] for initiating discussions of rejection and categoricity, respectively. This remark by Smiley [4, p.7] is especially noteworthy: 'If I am right, the practice of identifying a calculus with its consequence relation is only justified if specifying the consequence relation is sufficient to determine the entire sentential output of the semantics.' We endorse Smiley's view that the common practice of identifying calculi by using non-categorical consequence relations is a mistake.
} 
No acquaintance with the literature mentioned above is required to understand the results that we present.

\section{Multiple CONCLUSION CONSEQUENCE}

DEFINITION 1 Let $S$ be a set of sentences. $+s$ is an assertion on $\{+,-\} / S$ iff $s \in S$. $-s$ is a rejection on $\{+,-\} / S$ iff $s \in S$. $j$ is a judgment on $\{+,-\} / S$ iff $j$ is an assertion on $\{+,-\} / S$ or a rejection on $\{+,-\} / S$.

So, given sentences $a$ and $b$ the judgments on $\{+,-\} /\{a, b\}$ are $+a,-a,+b$, and $-b$. Judgments on $S$ are to be distinuished from sentences of $S$. And - is not a symbol for negation. ${ }^{2}$ Read $+a$ as ' $a$ is asserted.' Read $-a$ as 'a is rejected.'

DEFINITION 2 Suppose $J$ and $K$ are (empty or non-empty) sets of judgments on $\{+,-\} / S$. Then $\langle J, K\rangle$ is an inference on $\{+,-\} / S$.

DEFINITION 3 Let $S$ be a set of sentences. $v$ is a pr-valuation on $S$ iff $v$ is a subset of $S \times\{t, f\} .^{3}$ ('pr' is short for 'partial relation.')

DEFINITION 4 Let $v$ be a pr-valuation on $S$. Let $s$ be a member of $S$. $v$ satisfies $+s$ iff $\langle s, t\rangle \in v$, and $v$ satisfies $-s$ iff $\langle s, f\rangle \in v$. Let $\langle J, K\rangle$ be an inference on $\{+,-\} / S$. $v$ satisfies $\langle J, K\rangle$ iff $v$ does not satisfy some member of $J$ or $v$ satisfies some member of $K$.

DEFINITION 5 Let $V$ be a set of pr-valuations on $S$. Let $R$ be the set of inferences on $\{+,-\} / S$ such that $\langle J, K\rangle \in R$ iff every pr-valuation in $V$ satisfies $\langle J, K\rangle$. Then $R$ is a pr-consequence relation on $\{+,-\} / S$ and $R$ is the pr-consequence relation on $\{+,-\} / S$ generated by $V$.

DEFINITION 6 Suppose that $R$ is the pr-consequence relation on $\{+,-\} / S$ generated by a set $V$ of pr-valuations on $S$. $R$ is a categorical pr-consequence relation on $\{+,-\} / S$ iff there is no set $V^{\prime}$ of pr-valuations on $S$ such that $V \neq V^{\prime}$ and $R$ is the pr-consequence relation on $\{+,-\} / S$ generated by $V^{\prime}$.

Example 1. Suppose $a$ is a sentence. There are exactly 16 inferences on $\{+,-\} /\{a\}$, the subsets of $\{+a,-a\} \times\{+a,-a\}$. So there are exactly $2^{16}$ sets of inferences on $\{+,-\} /\{a\}$. There are exactly 4 pr-valuations on $\{a\}$, the subsets of $\{a\} \times\{t, f\}$. So there are exactly 16 sets of pr-valuations on $\{a\}$. So there are at most 16 pr-consequence relations on $\{+,-\} /\{a\}$.

We list the pr-valuations on $\{a\}: v_{1}=\{\langle a, t\rangle,\langle a, f\rangle\} ; v_{2}=\{\langle a, t\rangle\} ; v_{3}=$ $\{\langle a, f\rangle\} ;$ and $v_{4}=\emptyset$. Each of these pr-valuations does not satisfy an inference that is satisfied by the others as indicated by the following pairs: $v_{1},\langle\{+a,-a\}, \emptyset\rangle$; $v_{2},\langle\{+a\},\{-a\}\rangle ; v_{3},\langle\{-a\},\{+a\}\rangle ;$ and $v_{4},\langle\emptyset,\{+a,-a\}\rangle$. So there are exactly 16 pr-consequence relations on $\left.\{+,-\} \hat{a}_{\xi}\right\}_{\text {and }}$ each of them is categorical.

\footnotetext{
${ }^{2}$ Smiley $[4$, p. 1] says 'asserting not $P$ may be equivalent to rejecting $P$, but it is not the very same thing, any more than assserting $P$ and $Q$ is the same as the two assertions $P$ and $Q$.

${ }^{3}$ The notion of a pr-valuation is taken from Dunn [7].
}

$$
\{+,-3 /\{u\}
$$


THEOREM 7 Given any set $S$ of sentences, every pr-consequence relation on $\{+,-\} / S$ is categorical.

Proof: Let $S$ be any set of sentences and let $v$ be a pr-valuation on $S$. Let $\langle J, K\rangle$ be an inference on $\{+,-\} / S$ where $J \cup K$ is the universal set of judgments on $\{t,-\} / S, J \cap K=\emptyset$ and, for every sentence $s$ in $S,+s \in J$ iff $\langle s, t\rangle \in v$ and $-s \in J$ iff $\langle s, f\rangle \in v$. Then $v$ does not satisfy $\langle J, K\rangle$, but every valuation other than $v$ satisfies $\langle J, K\rangle$. So distinct sets of pr-valuations on $S$ generate distinct pr-consequence relations on $\{+,-\} / S$.

DEFINITION $8 v$ is a pf-valuation on a set $S$ of sentences iff $v$ is a pr-valuation on $S$ and for every sentence $s$ in $S$ either $\langle s, t\rangle \notin v$ or $\langle s, f\rangle \notin v . v$ is a tr-valuation on $S$ iff $v$ is a pr-valuation on $S$ and for every sentence $s$ in $S$ either $\langle s, t\rangle \in v$ or $\langle s, f\rangle \in v . v$ is a tf-valuation on $S$ iff $v$ is a pf-valuation on $S$ and a tr-valuation on $S$. ('pf,' 'tr,' and 'tf' are short for 'partial function,' 'total relation,' and 'total function,' respectively.)

Imitate Definitions 5 and 6 to define pf-, tr-, and tf- consequence relations and categorical pf-, tr-, and tf- consequence relations.

Example 2. Suppose $a$ is the only sentence. Though $\{\langle a, t\rangle,\langle a, f\rangle\}$ is a trvaluation it is not a pf-valuation and thus not a tf-valuation. Though $\emptyset$ is a pfvaluation it is not a tr-valuation and thus not a tf-valuation. There are exactly 8 sets of pf-valuations, exactly 8 sets of tr-valuations and exactly 4 sets of tf-valuations on $\{a\}$. Counts for the pf-, tr- and tf- consequence relations on $\{+,-\} /\{a\}$ are 8 , 8 , and 4 , respectively. The pf- (tr-, tf-) consequence relations on $\{+,-\} / a$ are categorical pf- (tr-, tf-) consequence relations on $\{+,-\} / a$.

THEOREM 9 Every pf- (tr-, tf-) consequence relation on $\{+,-\} / S$ is a categorical pf- (tr-, tf-) consequence relation on $\{+,-\} / S .^{4}$

Proof: Use Theorem 1 .

Suppose we exclude rejections or exclude assertions.

THEOREM 10 Every tf-consequence relation on $\{+\} / S$ is categorical, and every tf-consequence relation on $\{-\} / S$ is categorical.

Proof: Note that a tf-valuation on $S$ satisfies $\langle+J \cup-K,+L \cup-M\rangle$ iff it satisfies $\langle+J \cup+M,+K \cup+L\rangle$ iff it satisfies $\langle-K \cup-L,-J \cup-M\rangle$.

Rumfitt [1] points out for some $S$ there are pf-consequence relations on $\{+\} / S$ that are not categorical. We show this by using a simple example. Let $S=\{a\}$. The pf-consequence on $\{+\} / a$ relation generated by the set $\{\{\langle a, t\rangle\},\{\langle a, f\rangle\}$ of pf-valuations on $\{a\}$ is the pf-consequence relation on $\{+\} / a$ generated by the set $\{\{\langle a, t\rangle\},\{\langle a, f\rangle\}, \emptyset\}$ of pf-valuations on $\{a\}$.

\footnotetext{
${ }^{4}$ Rumfitt[1] proves that pf-consequence relations on $\{t,-\} / S$ are categorical.
} 
Note also that for some $S$ there are tr-consequence relations on $\{+\} / S$ that are not categorical. Let $S=\{a\}$. The tr-consequence relation on $\{+\} / a$ generated by the set $\{\{\langle a, t\rangle\},\{\langle a, f\rangle\}\}$ of tr-valuations on $\{a\}$ is the $\operatorname{tr}$-consequence relation on $\{+\} / a$ generated by the set $\{\{\langle a, t\rangle\},\{\langle a, f\rangle\},\{\langle a, t\rangle,\langle a, f\rangle\}\}$ of tr-valuations on $\{a\}$.

Given either of these results there are pr-consequence relations on $\{+\} /\{a\}$ that are not categorical.

By referring to the above sets of valuations one can easily show that there are pf-, tr- and pr-consequence relations on $\{-\} /\{a\}$ that are not categorical.

\section{MULTIPLE CONCLUSION DEDUCIBILITY}

DEFINITION 11 Let $J$ be a set of judgments on $\{+,-\} / S .\left\langle J_{1}, J_{2}\right\rangle$ is a partition of $J$ iff $J_{1} \cup J_{2}=J$ and $J_{1} \cap J_{2}=\emptyset$.

DEFINITION 12 A set $R$ of inferences on $\{+,-\} / S$ is an $O D C$-deducibility relation on $\{+,-\} / S$ iff it meets the following conditions:

i) (Overlap) If $J$ and $K$ are sets of judgments on $\{+,-\} / S$, then if $J \cap K \neq \emptyset$ then $\langle J, K\rangle \in R$;

ii) (Dilution) If $J^{\prime}$ and $K^{\prime}$ are sets of judgments on $\{+,-\} / S$, then if $J \subseteq J^{\prime}$, $K \subseteq K^{\prime}$, and $\langle J, K\rangle \in R$ then $\left\langle J^{\prime}, K^{\prime}\right\rangle \in R$; and

iii) (Cut) If $J, K$ and $L$ are sets of judgments on $\{+,-\} / S$, then if, for every partition $\left\langle L_{1}, L_{2}\right\rangle$ of $L,\left\langle J \cup L_{1}, L_{2} \cup K\right\rangle \in R$ then $\langle J, K\rangle \in R$.

So, for example, the universal set $U$ of inferences on $\{+,-\} /\{a\}$ is an ODCdeducibility relation on $\{+,-\} /\{a\}$. But $U-\{\langle\emptyset, \emptyset\rangle\}$ is not. Though the latter set meets the Overlap and Dilution conditions it does not meet the Cut condition since $\langle\emptyset,\{+a\}\rangle$ and $\langle\{+a\}, \emptyset\rangle$ belong to the set but $\langle\emptyset, \emptyset\rangle$ does not. $U-\{\langle\emptyset,\{+a,-a\}\rangle\}$ is not an ODC-deducibility relation on $\{+,-\} /\{a\}$. It meets the Overlap and Cut conditions but does not meet the Dilution condition since $\langle\emptyset, \emptyset\rangle$ belongs to it but $\langle\emptyset,\{+a,-a\}\rangle$ does not. $\{\langle\{+a,-a\},\{+a,-a\}\rangle\}$ is not an ODC-deducibility relation on $\{+,-\} /\{a\}$. It meets the Dilution and Cut conditions but does not meet the Overlap condition since $\langle\{+a,+a\}\rangle$ does not belong to it.

THEOREM 13 [Generalized soundness] Every pr-consequence relation on $\{+,-\} / S$ is an ODC-deducibility relation on $\{+,-\} / S$.

Proof: Suppose $R$ is a pr-consequence relation on $\{+,-\} / S$ generated by a set $V$ of pr-valuations on $S$. Suppose $J$ and $K$ are sets of judgments on $\{+,-\} / S$. Overlap. Suppose $J \cap K \neq \emptyset$. Then for some sentence $a$ either $+a \in J \cap K$ or $-a \in J \cap K$. If the former holds then $\langle J, K\rangle \in R$ since, for every valuation $v$ in $V$, $\langle a, t\rangle \in v$ or $\langle a, t\rangle \notin v$. If the latter holds then $\langle J, K\rangle \in R$ since, for every valuation $v$ in $V\langle a, f\rangle \in v$ or $\langle a, f\rangle \notin v$. Dilution. Suppose $J^{\prime}$ and $K^{\prime}$ are sets of judgments on $\{+,-\} / S, J \subseteq J^{\prime}$, and $K \subseteq K^{\prime}$. Suppose $\left\langle J^{\prime}, K^{\prime}\right\rangle \notin R$. Then there is a valuation $v$ in $V$ that does not satisfy $\left\langle J^{\prime}, K^{\prime}\right\rangle$. Then $v$ does not satisfy $\langle J, K\rangle$. Cut. Suppose $L$ is a set of judgments on $\{+,-\} / S$. Given any pr-valuation $v$ on $S$ we construct 
a partition $\left\langle L_{1}, L_{2}\right\rangle$ of $L$ such that $v$ does not satisfy it. Suppose $+a \in L$. Put $+a$ in $L_{1}$ iff $\langle a, t\rangle \in v$. Suppose $-a \in L$. Put $-a$ in $L_{1}$ iff $\langle a, f\rangle \in v$. So, if $\langle J, K\rangle \notin R$ then $\left\langle J \cup L_{1}, L_{2} \cup K\right\rangle \notin R$.

THEOREM 14 [Generalized completeness] Every ODC-deducibility relation on $\{+,-\} / S$ is a pr-consequence relation on $\{+,-\} / S$.

Proof: Let $R$ be an ODC-deducibility relation on $\{+,-\} / S$. We identify a prconsequence relation $\vDash_{V}$ on $\{+,-\} / S$ such that $R=F_{V}$. Let $v_{\left\langle U_{1}, U_{2}\right\rangle}$ be the pr-valuation that does not satisfy $\left\langle U_{1}, U_{2}\right\rangle$, where $\left\langle U_{1}, U_{2}\right\rangle$ is a partition of the universal set of judgments. Let $V=\left\{v_{\left\langle U_{1}, U_{2}\right\rangle} \mid\left\langle U_{1}, U_{2}\right\rangle \notin R\right\}$. Let $\vDash_{V}$ be the pr-consequence relation generated by $V$.

$R \subseteq \vDash V$. Suppose $\langle J, K\rangle \notin \vDash V$. Then, for some partition $\left\langle U_{1}, U_{2}\right\rangle$ of the universal set of judgments, $v_{\left\langle U_{1}, U_{2}\right\rangle}$ does not satisfy $\langle J, K\rangle$ and $\left\langle U_{1}, U_{2}\right\rangle \notin R$. Suppose $\langle J, K\rangle \in R$. If $J \subseteq U_{1}$ and $K \subseteq U_{2}$, by Dilution, $\left\langle U_{1}, U_{2}\right\rangle \in R$. Otherwise, $v_{\left\langle U_{1}, U_{2}\right\rangle}$ satisfies $\langle J, K\rangle$. So $\langle J, K\rangle \notin R$.

$\vDash_{V} \subseteq R$. Suppose $\langle J, K\rangle \notin R$. By Cut there is a partition $\left\langle U_{1}, U_{2}\right\rangle$ of the universal set of judgments such that $\left\langle J \cup U_{1}, U_{2} \cup K\right\rangle \notin R$. By Overlap $J \subseteq U_{1}$ and $K \subseteq U_{2}$. So $\left\langle U_{1}, U_{2}\right\rangle \notin R$. So $v_{\left\langle U_{1}, U_{2}\right\rangle} \in V$. Since $v_{\left\langle U_{1}, U_{2}\right\rangle}$ does not satisfy $\left\langle U_{1}, U_{2}\right\rangle, v_{\left\langle U_{1}, U_{2}\right\rangle}$ does not satisfy $\langle J, K\rangle$. So $\langle J, K\rangle \notin \vDash V$.

DEFINITION $15 R$ is an ODCE-deducibility relation on $\{+,-\} / S$ iff $\mathrm{R}$ is an ODC-deducibility relation that meets this condition: (ex falso quodlibet) for every sentence $s$ in $S,\langle\{+s,-s\}, \emptyset\rangle \in R . R$ is an ODCT-deducibility relation on $\{+,-\} / S$ iff $R$ is an ODC-deducibility relation that meets this condition: (tertium non datur) for every sentence $s$ in $S,\langle\emptyset,\{+s,-s\}\rangle \in R$. $R$ is an ODCET-deducibility relation on $\{+,-\} / S$ iff $\mathrm{R}$ is an ODCE-deducibility relation on $\{+,-\} / S$ and an ODCTdeducibility relation on $\{+,-\} / S$.

THEOREM 16 A set of inferences is a pf-consequence relation on $\{t,-\} / S$ iff it is an ODCE-deducibility relation on $\{+,-\} / S$.

Proof: Soundness. Every pf-consequence relation on $\{+,-\} / S$ is a pr-consequence relation on $\{+,-\} / S$. So, given Theorem 4 , it suffices to show that every pfconsequence relation on $\{t,-\} / S$ meets the ex falso quodlibet condition. Suppose $\mathrm{R}$ is a pf-consequence relation on $\{+,-\} / S$ generated by a set $V$ of pf-valuations on $S$. Suppose some member $v$ of $V$ does not satisfy $\langle\{+s,-s\}, \emptyset\rangle$ for some sentence $s$ in $S$. Then $\langle s, t\rangle \in v$ and $\langle s, f\rangle \in v$. Then $v$ is not a pf-valuation on $S$.

Completeness. Imitate the reasoning for Theorem 5 . Let $\left\langle U_{1}, U_{2}\right\rangle$ range over partitions of the universal set of judgments on $\{+,-\} / S$ that meet this condition (E): for every sentence $s$ in $S$ either $+s \notin U_{1}$ or $-s \notin U_{1}$.

THEOREM 17 A set of inferences is a tr-consequence relation on $\{+,-\} / S$ iff it is an ODCT-deducibility relation on $\{+,-\} / S$. 
Proof: Soundness. Every tr-consequence relation on $\{+,-\} / S$ is a pr-consequence relation on $\{t,-\} / S$. So, given Theorem 4 , it suffices to show that every trconsequence relation on $\{+,-\} / S$ meets the tertium non datur condition. Suppose $\mathrm{R}$ is a tr-consequence relation on $\{+,-\} / S$ generated by a set $V$ of tr-valuations on $S$. Suppose some member $v$ of $V$ does not satisfy $\langle\emptyset,\{+s,-s\}\rangle$ for some sentence $s$ in $S$. Then $\langle s, t\rangle \notin v$ and $\langle s, f\rangle \notin v$. Then $v$ is not a tr-valuation on $S$.

Completeness. Imitate the reasoning for Theorem 5 . Let $\left\langle U_{1}, U_{2}\right\rangle$ range over partitions of the universal set of judgments on $\{+,-\} / S$ that meet this condition $(\mathrm{T})$ : for every sentence $s$ in $S$ either $+s \notin U_{2}$ or $-s \notin U_{2}$.

THEOREM 18 A set of inferences is a tf-consequence relation on $\{+,-\} / S$ iff it is an ODCET-deducibility relation on $\{+,-\} / S$.

Proof: Soundness. Note that every tf-consequence relation on $\{+,-\} / S$ is a pf-consequence relation on $\{+,-\} / S$ and a tr-consequence relation on $\{+,-\} / S$.

Completeness. Imitate the reasoning for Theorem 5. Let $\left\langle U_{1}, U_{2}\right\rangle$ range over partitions of the universal set of judgments on $\{+,-\} / S$ that meet conditions $\mathrm{E}$ and $\mathrm{T}$.

Proofs for the following three theorems are given by modifying preceding reasoning.

THEOREM 19 A set of inferences is a tf-consequence relation on $\{+\} / S$ iff it is an ODC-deducibility relation on $\{+\} / S$.

THEOREM 20 A set of inferences is a tf-consequence relation on $\{-\} / S$ iff it is an ODC-deducibility relation on $\{-\} / S$.

Define consequence relations on $\emptyset / S$ in the natural way. Premise and conclusion sets are sets of sentences, not sets of judgments.

THEOREM 21 [Shoesmith and Smiley] A set of inferences is is a tf-consequence relation on $\emptyset / S$ iff it is an ODC-deducibility relation on $\emptyset / S$.

\section{Single CONClusion CONSEQUence}

Following Johnson [2] we formalize two notions of assertion and two notions of rejection. In addition to the notions of "strong assertion" and "strong rejection" discussed above we recognize "weak assertion", which complements strong rejection, and "weak rejection", which complements strong assertion."

\footnotetext{
${ }^{5}$ Rumfitt's [1] informal discussion of the the two rejection activities uses "internal" instead of "strong" and "external" instead of "weak." Rumfitt says that one who "rejects as false" the claim that the King of France is bald internally [strongly] rejects the claim, but one who "rejects as not true" the claim that the King of France is bald externally [weakly] rejects the claim. Rumfitt mentions the two kinds of rejection only to indicate that his focus is on what we are calling strong rejection. Since Rumfitt confined his discussion of categorical consequence relations to the multiple conclusion, partial function variety he had no need to formalize the two kinds of rejection and the two kinds of assertion.
} 
DEFINITION 22 Let $S$ be a set of sentences. Let $s$ be a member of $S+s(\oplus s,-s$, $\Theta s$ ) is a strong assertion (a weak assertion, a strong rejection, a weak rejection) on $\{+, \oplus,-, \ominus\} / S . j$ is a judgment on $\{+, \oplus,-, \ominus\} / S$ iff $j$ is a strong assertion, a weak assertion, a strong rejection, or a weak rejection on $\{+, \oplus,-, \theta\} / S$.

DEFINITION 23 Suppose $J$ is a set of judgments and $k$ is a judgment on $\{+, \oplus,-$, $\ominus\} / S$. Then $\langle J, k\rangle$ is an sc-(single conclusion) inference on $\{+, \oplus,-, \Theta\} / S$.

DEFINITION 24 Let $v$ be a pr-valuation on $S$. Let $s$ be a member of $S$. $v$ satisfies $\oplus s$ iff $\langle s, f\rangle \notin v$ and $v$ satisfies $\ominus s$ iff $\langle s, t\rangle \notin v$. Let $\langle J, k\rangle$ be an sc-inference on $\{+, \oplus,-, \ominus\} / S$. $v$ satisfies $\langle J, k\rangle$ iff $v$ does not satisfy some member of $J$ or $v$ satisfies $k$.

Extend the other notions defined for multiple conclusion inferences to single conclusion inferences in the natural way.

Example 3. Suppose $S=\{a\}$. There are exactly 256 sc-inferences on $\{+, \oplus,-, \ominus\} /\{a\}$. Let the pr-valuations on $\{a\}$ be defined as in Example 1. Each of these pr-valuations does not satisfy an sc-inference on $\{+, \oplus,-, \ominus\} /\{a\}$ that is satisfied by the others as indicated by the following pairs: $v_{1},\langle\{+a\}, \oplus a\rangle, v_{2},\langle\{+a\},-a\rangle$, $v_{3},\langle\{-a\},+a\rangle$, and $v_{4},\langle\{\oplus a\},+a\rangle$. So there are exactly 16 sc-pr-consequence relations on $\{+, \oplus,-, \Theta\} /\{a\}$ and each of them is categorical.

THEOREM 25 Every sc-pr-consequence relation on $\{+, \oplus,-, \ominus\} / S$ is categorical.

Proof: For every pr-valuation $v$ on $S$ we construct an sc-inference $\langle J-\{k\}, k\rangle$ on $\{+, \oplus,-, \Theta\} / S$ such that $v$ does not satisfy it but every other pr-valuation on $S$ does. Pick a sentence $a$ that belongs to $S$. If $\langle a, t\rangle \in v$ let $k$ be $\ominus a$, otherwise let $k$ be $+a$. If $\langle a, f\rangle \in v$ put $-a$ in $J-\{k\}$, otherwise put $\oplus a$ in $J-\{k\}$. For every sentence $b$ other than $a$ in $S:$ i) if $\langle b, t\rangle \in v$ put $+b$ in $J-\{k\}$, otherwise put $\Theta b$ in $J-\{k\}$; and ii) if $\langle b, f\rangle \in v$ put $-b$ in $J-\{k\}$, otherwise put $\oplus b$ in $J-\{k\}$.

COROLLARY 26 Every sc-pf-, sc-tr-, and sc-tf-consequence relation on $\{+, \oplus,-, \theta\} / S$ is categorical. ${ }^{6}$

Note that if $F$ is a three-membered subset of $\{+, \oplus,-, \Theta\}$ we can choose $S$ so that there are sc-pf- and thus sc-pr consequence relations on $F / S$ that are not categorical. The pf-consequence relation on $\{-, \oplus, \ominus\} /\{a, b\}$ generated by $\{\{\langle a, t\rangle\},\{\langle b, t\rangle\}\}$ is the pf-consequence relation on $\{-, \oplus, \ominus\} /\{a, b\}$ generated by $\{\{\langle a, t\rangle\},\{\langle b, t\rangle\},\{\langle a, t\rangle,\langle b, t\rangle\}\}$. For $\{+, \oplus, \Theta\} /\{a, b\}$ use $\{\{\langle a, f\rangle\},\{\langle b, f\rangle\}\}$ and $\{\{\langle a, f\rangle\},\{\langle b, f\rangle\},\{\langle a, f\rangle,\langle b, f\rangle\}\}$. For $\{+,-, \ominus\} /\{a, b\}$ use $\{\{\langle a, f\rangle\}$, $\{\langle b, f\rangle\}\}$ and $\{\{\langle a, f\rangle\},\{\langle b, f\rangle\}, \emptyset\}$. For $\{+,-, \oplus\} /\{a, b\}$ use $\{\{\langle a, t\rangle\},\{\langle b, t\rangle\}\}$ and $\{\{\langle a, t\rangle\},\{\langle b, t\rangle\}, \emptyset\}$.

Likewise, if $F$ is a three-membered subset of $\{+, \oplus,-, \theta\}$ there are sc-tr-consequence relations on $F /\{a, b\}$ that are not categorical.

\footnotetext{
${ }^{6}$ Johnson [2] proves that every sc-pf-consequence relation on $\{+, \oplus,-, \ominus\} / S$ is categorical.
} 
THEOREM 27 Let $F$ be a two-membered subset of $\{+, \oplus,-, \theta\}$ where one member is + or $\oplus$ and the other member is - or $\Theta$. Every sc-tf-consequence relation on $F / S$ categorical. $^{7}$

Proof: For every tf-valuation $v$ on $S$ we construct an sc-inference $\langle J-\{k\}, k\rangle$ on $\{+(\oplus),-(\Theta)\} / S$ such that $v$ does not satisfy it but every other pr-valuation on $S$ does. Pick a sentence $a$ that belongs to $S$. If $\langle a, t\rangle \in v$ let $k$ be $-a(\Theta a)$, otherwise let $k$ be $+a(\oplus a)$. For every sentence $b$ other than $a$ in $S$ if $\langle b, t\rangle \in v$ put $+b(\oplus b)$ in $J-\{k\}$, otherwise put $-b(\ominus b)$ in $J-\{k\}$.

\section{Single CONCLUSION DEDUCIBILITY}

DEFINITION 28 Let $J$ be a set of judgments on $\{+, \oplus,-, \ominus\} / S . J$ is an fc (full and consistent) set of judgments relative to a set $A$ of sentences $(A \subseteq S$ ) iff: i) for each sentence $s$ in $A$ there are exactly two judgments with $s$ as content that belong to $J$; ii) neither of these judgments is an opposite of the other; and iii) the only judgments in $J$ are those whose content is a member of $A . s$ is the content of $+s$, $\oplus s,-s$ and $\ominus s$. The opposite of $+s$ is $\Theta s$. In symbols $o p(+s)=\ominus s . o p(\ominus s)=+s$, $o p(-s)=\oplus s$. And $o p(\oplus s)=-s$.

DEFINITION 29 Let $R$ be a set of sc-inferences on $\{+, \oplus,-, \theta\} / S$. $R$ is an $s c$ $O D C R$ deducibility relation on $\{+, \oplus,-, \ominus\} / S$ iff $R$ meets the following conditions:

i) (sc-overlap) If $k \in J$ then $\langle J, k\rangle \in R$;

ii) (sc-dilution) If $J \subseteq J^{\prime}$ and $\langle J, k\rangle \in R$ then $\left\langle J^{\prime}, k\right\rangle \in R$;

iii) (sc-cut) If $\langle J \cup K, k\rangle \in R$ for every fc set $K$ of judgments relative to $A(A \subseteq S)$ then $\langle J, k\rangle \in R$; and

iv) (sc-reversal) If $\langle J \cup\{j\}, k\rangle \in R$ then $\langle J \cup\{o p(k)\}, o p(j)\rangle \in R$.

The following derived rule, Opposites, will be used below.

Let $R$ be an sc-ODCR deducibility relation on $\{+, \oplus,-, \ominus\} / S$. Opposites: If $\{j, o p(j)\} \subseteq J$ then, for every judgment $k,\langle J, k\rangle \in R$.

Proof: Assume the antecedent. Let $J=J^{\prime} \cup\{j, o p(j)\}$. By sc-overlap $\left\langle J^{\prime} \cup\right.$ $\{j, o p(j), o p(k)\}\rangle j \in R$. By sc-reversal $\left\langle J^{\prime} \cup\{j, o p(j)\}, k\right\rangle \in R$. So $\langle J, k\rangle \in R$.

THEOREM 30 Every sc-pr consequence relation on $\{+, \oplus,-, \ominus\} / S$ is an sc-ODCR deducibility relation on $\{+, \oplus,-, \Theta\} / S$.

Proof: Let $v$ be a pr-valuation on $S$. sc-overlap. For each judgment on $\{+, \oplus,-, \ominus\} / S$ with content $s,\langle s, t\rangle$ does or does not belong to $v$ and $\langle s, f\rangle$ does or does not belong to $v$. sc-dilution. Suppose $J \subseteq J^{\prime}$. Note that if $v$ satisfies every member of $J^{\prime}$ then $v$ satisfies every member of $J$. sc-cut. Suppose $v$ does not satisfy $\langle J, k\rangle$. Let $A$ be any set of sentences. Let $K$ be an fc set of judgments relative to $A$ such that for each sentence $s$ in $A+s(-s, \oplus s, \Theta) \in K$ iff $\langle s, t\rangle \in v(\langle s, f\rangle \in v$, $\langle s, f\rangle \notin v,\langle s, t\rangle \notin v)$, respectively. Then $v$ does not satisfy $\langle J \cup K, k\rangle$. sc-reversal. Suppose $v$ does not satisfy $\langle J \cup\{o p(k)\}, o p(j)\rangle$. Then $v$ satisfies $j$ but does not

\footnotetext{
${ }^{7}$ Smiley [4] proves that every sc-tf-consequence relation on $\{+,-\} / S$ is categorical.
} 
satisfy $k$. So $v$ does not satisfy $\langle J \cup j, k\rangle$.

THEOREM 31 Every sc-ODCR deducibility relation on $\{+, \oplus,-, \ominus\} / S$ is an scpr-consequence relation on $\{+, \oplus,-, \ominus\} / S$.

Proof: Let $R$ be an sc-ODCR deducibility relation on $\{+, \oplus,-, \ominus\} / S$. Let $I=$ $\langle U, m\rangle$ where $m \notin U$ and $U$ is an fc set of judgments relative to $S$ on $\{+, \oplus,-, \ominus\} / S$. Let $v_{I}$ be the pr-valuation that does not satisfy $I$. Let $V=\left\{v_{I} \mid I \notin R\right\}$. Let $\models_{V}$ be the pr-consequence relation generated by $V$. Then $R=\vDash V$.

$R \subseteq \vDash_{V}$. Suppose $\langle J, k\rangle \notin \vDash V$. Then, for some $v_{I}$ in $V(I=\langle U, m\rangle), v_{I}$ does not satisfy $\langle J, k\rangle$. Since $V_{I}$ satisfies $U, J \cup\{o p(k)\} \subseteq U$. Suppose $\langle J, k\rangle \in R$. By sc-dilution $\langle J \cup U \cup\{o p(m)\}, k\rangle \in R$. By sc-reversal $\langle J \cup\{o p(k)\} \cup U\}, m\rangle \in R$. Then $\langle U, m\rangle \in R$. But $\langle U, m\rangle \notin R$. So $\langle J, k\rangle \notin R$.

$\vDash_{V} \subseteq R$. Suppose $\langle J, k\rangle \notin R$. By sc-cut $\langle J \cup U, k\rangle \notin R$, where $U$ is an fc set of judgments relative to $S$ on $\{+, \oplus,-, \ominus\} / S$. By sc-overlap $k \notin U$. By the derived rule Opposites, $J \subseteq U$. So $v_{\langle U, k\rangle} \in V$. Since $v_{\langle U, k\rangle}$ does not satisfy $\langle U, k\rangle, v_{\langle U, k\rangle}$ does not satisfy $\langle J, k\rangle$. So $\langle J, k\rangle \notin \vDash V$.

DEFINITION $32 R$ is an sc-ODCRE deducibility relation on $\{+,-, \oplus, \ominus\} / S$ iff $R$ is an sc-ODCR deducibility relation on $\{+,-, \oplus, \ominus\} / S$ that meets this condition: for every sentence $s$ in $S,(\{+s\}, \oplus s\rangle \in R$. $R$ is an sc-ODCRT deducibility relation on $\{+,-, \oplus, \ominus\} / S$ iff $R$ is an sc-ODCR deducibility relation on $\{+,-, \oplus, \ominus\} / S$ that meets this condition: for every sentence $s$ in $S,\langle\{\oplus s\},+s\rangle \in R . R$ is an sc-ODCRET deducibility relation on $\{+,-, \oplus, \ominus\} / S$ iff $R$ is an sc-ODCRE deducibility relation on $\{+,-, \oplus, \ominus\} / S$ and an sc-ODCRT deducibility relation on $\{+,-, \oplus, \ominus\} / S$.

THEOREM 33 A set of inferences is an sc-pf- (sc-tr-, sc-tf-) consequence relation on $\{+,-, \oplus, \ominus\} / S$ iff it is an sc-ODCRE (sc-ODCRT, sc-ODCRET) deducibility relation on $\{+,-, \oplus, \ominus\} / S$.

Proof: Imitate the proofs of the preceding two theorems.

\section{REFERENCES}

[1] I. Rumfitt. The categoricity problem and truth-value gaps. Analysis, 57:223$235,1997$.

[2] Fred Johnson. Categoricity of partial logics. In Timothy Childers, editor, The Logica Yearbook 1998, pages 194-202. Filosofia, Prague, 1999.

[3] D.J. Shoesmith and T. J. Smiley. Multiple-conclusion logic. Cambridge University Press, 1978.

[4] T. J. Smiley. Rejection. Analysis, 56:1-9, 1996. 
[5] Rudolf Carnap. Introduction to Semantics. Harvard University Press, 1942.

[6] Rudolf Carnap. Formalization of logic. Harvard University Press, 1943.

[7] J.M. Dunn. Intuitive semantics for first degree entailment and 'coupled trees'. Philosophical Studies, 29:149-168, 1976. 